\title{
New physics at the FCC-ee: indirect discovery potential
}

\author{
Jorge de Blas ${ }^{1,2, a}$ (D) \\ ${ }^{1}$ CAFPE and Departamento de Física Teórica y del Cosmos, Universidad de Granada, 18071 Granada, \\ Spain \\ 2 Institute of Particle Physics Phenomenology, Durham University, Durham DH1 3LE, UK
}

Received: 8 April 2021 / Accepted: 7 August 2021

(C) The Author(s) 2021, corrected publication 2022

\begin{abstract}
We review the projected sensitivity to physics beyond the Standard Model via indirect searches at the Future $e^{+} e^{-}$Circular Collider (FCC-ee). The indirect sensitivity to new physics is discussed both from a model-independent perspective, using the formalism of Effective Field Theories, but also from the point of view of more specific classes of well-motivated models.
\end{abstract}

\section{Introduction}

The experiments at the Large Hadron Collider (LHC) as well as from the past Large Electron Positron collider (LEP) and Stanford Linear Collider (SLC) provided the first detailed look at the properties of the Higgs and Electroweak (EW) gauge bosons, respectively. The implications of these measurements are of profound significance, not only validating the Standard Model (SM) description of most of the particle physics phenomena known today, but also imposing severe constraints on models with new physics (NP). And yet, we have both phenomenological evidence that such physics beyond the SM (BSM) must exist, as well as compelling theoretical reasons to believe that some form of NP should not be dramatically heavier than the EW scale. Indeed, phenomenological observations such as neutrino oscillations, dark matter, or the matter-antimatter asymmetry cannot be successfully explained with SM physics. On the theory side, solutions to the gauge hierarchy problem, i.e. why the EW scale is stable in presence of other high-energy scales of nature, typically require that some form of new phenomena arises around the TeV. In this article, we focus on the potential of the FCC-ee to learn from BSM physics via indirect probes, i.e. studying SM processes and looking for deviations with respect to the SM predictions, which could be attributed to NP effects [1]. The FCC-ee design [2] is capable of delivering high-precision measurements of processes involving the whole SM spectrum: from the Higgs and the EW gauge bosons, to the fermionic particles, including the Top quark. It is in this sense a full SM-particle factory and, via the measurements of the SM properties - in most cases with an order of magnitude

This work has been supported in part by the FEDER/Junta de Andalucía Project Grant P18-FRJ-3735 and by the UK Science and Technology Facilities Council (STFC) Grant ST/P001246/1. Funding for open access charge: Universidad de Granada / CBUA.

\footnotetext{
a e-mail: jdeblasm@gmail.com (corresponding author)
} 
improvement with respect to the knowledge that will be possible at the High-Luminosity LHC (HL-LHC) - an ideal machine for these type of indirect tests.

The main challenge to be faced in the search of NP at the FCC-ee lies on the nonobservation of clear evidence of NP at the LHC. No BSM particles have been found and, with few exceptions such as the recent anomalies in $B$ physics, all current tests of the EW sector are consistent with the SM predictions. In other words, there is no specific guidance pointing towards the exploration of a particular class of NP scenarios. A possible solution to this problem is to approach the search in a model-independent way, using the formalism of Effective Field Theories (EFT). These provide a guiding rationale to organize NP corrections to SM processes, and to map these into a broad class of scenarios under minimal assumptions. The so-called Standard Model Effective Field Theory (SMEFT) has been used as a common framework for the assessment of the future collider capabilities in terms of indirect tests of NP, applicable to models where new particles are heavy compared to the EW scale. In the first two sections of this article we will review the status of such SMEFT studies and the implications for several classes of NP models.

One must also note, however, that, while no new particles have been directly observed at the LHC, the possible existence of light degrees of freedom associated with well-motivated BSM scenarios is not completely excluded by current data. Therefore, while a model-independent approach seems to be a sensible way to assess the capabilities of future machines such as the FCC-ee, it is also important to frame such physics potential studies in terms of more specific scenarios that could be of theoretical or phenomenological interest. The important question is then, How can the FCC-ee measurements better tests these type of models compared to the LHC? This will be briefly addressed in Sect. 4.

\section{Effective field theory exploration of new physics at the FCC-ee}

In the SMEFT, the SM Lagrangian is extended with an infinite tower of operators of canonical mass dimension $d>4, \mathcal{O}_{i}^{(d)}$, constructed only with the SM degrees of freedom and symmetries. For heavy NP satisfying the decoupling theorem, the infinite series can be ordered according to the operator mass dimension:

$$
\mathcal{L}_{\mathrm{SMEFT}}=\mathcal{L}_{\mathrm{SM}}+\sum_{d>4} \frac{1}{\Lambda^{d}} \sum_{i} c_{i}^{(d)} \mathcal{O}_{i}^{(d)}=\mathcal{L}_{\mathrm{SM}}+\frac{1}{\Lambda^{2}} \sum_{i} c_{i} \mathcal{O}_{i}+\ldots,
$$

where $\Lambda$ is the EFT cut-off scale, and $d$-dimensional operator effects are suppressed by $(E / \Lambda)^{d-4}$, with $E \ll \Lambda$ the typical energy scale of a process. In the second equality we have assumed $B$ and $L$ conservation, in which case the leading BSM effects are given by dimension- 6 operators. ${ }^{1}$ These induce not only deformations in the different SM interactions, e.g. the standard $Z$ or Higgs couplings, but also include new tensor structures not present in the SM, e.g. anomalous triple gauge couplings (aTGC). The projections for the FCC-ee sensitivity to such deformations in the SMEFT formalism have been recently reviewed in $[3,4]$, and are shown in Fig. 1, from a global-fit study combining the EW precision observables (EWPO) at the $Z$-pole, and the $W W$ and Higgs measurements. The figure compares the FCCee reach with the information that will be available at the HL-LHC. The results show how the future Higgs factory would reach the per-mile level precision for some of the Higgs interactions, while the FCC-ee EWPO would bring precisions at the $10^{-5}$ level for the EW

1 Otherwise, there is one $L$-violating operator at dimension 5. This can generate Majorana neutrino masses but has otherwise no impact in the precision measurements discussed here. 

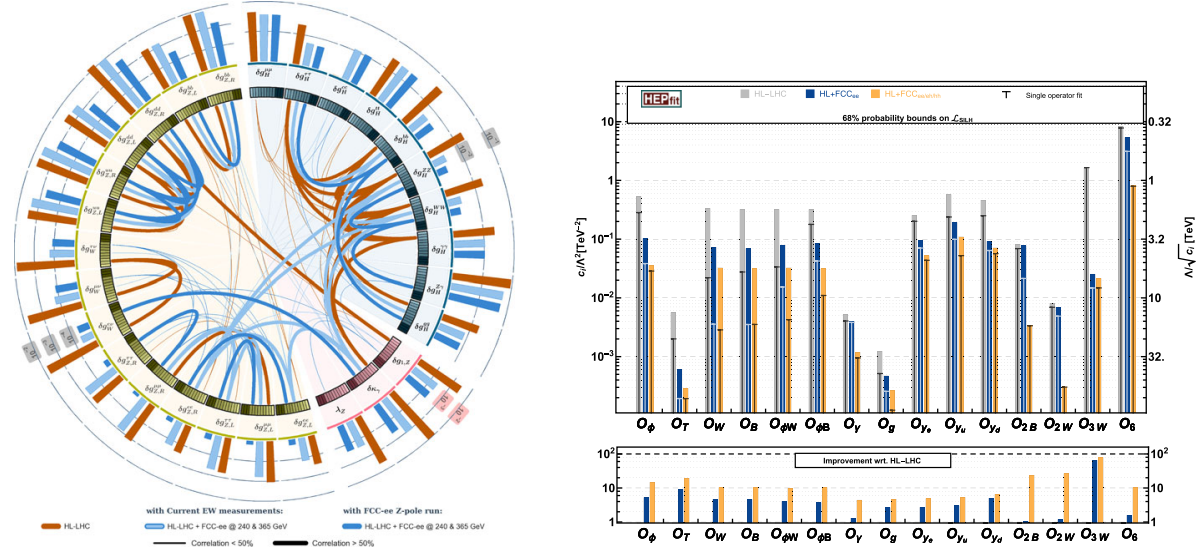

Fig. 1 (Left) Adapted from [4]: sensitivity at 68\% probability to deviations in the different EW/H/aTGC interactions from the SMEFT fit at the HL-LHC and HL-LHC + FCC-ee, with and without the FCC-ee Z-pole run. Lines connecting each pair of couplings indicate the strength of the correlation. (Right) 68\% probability reach for the Wilson coefficients $c_{i} / \Lambda^{2}$ from a fit to the EFT Lagrangian in Eq. (3.19) in Ref. [3]. The right axis shows the corresponding bound on the NP interaction scale

ones. The figure also illustrates the relevance of the $Z$-pole run in the determination of the Higgs properties, by removing the correlations between the EW and Higgs couplings. Of course, all these projections also rely on the non-trivial effort from the theory side to reduce the uncertainty of the SM calculations to a level comparable to or below the experimental one [5]. This will be particularly challenging for the calculation of the EWPO, where the full 3-loop and even the leading 4-loop effects may be needed.

Not shown in the figure are the FCC-ee capabilities to test the Top quark sector. The events collected at the $t \bar{t}$ threshold and the $365 \mathrm{GeV}$ run bring clear physics opportunities in testing NP modifying the EW interactions of the 3rd family of up-type quarks. As shown in [6], an optimal observable analysis would be sensitive to $\delta g_{Z, L(R)}^{t t}$ at the percent level. It is here, however, where the FCC-ee faces one of the most important challenges. As the tests of the $Z t t$ couplings are performed away from the $Z$ pole, a fully model-independent exploration of these interaction in the SMEFT framework requires to consider the effects of other operators not directly related to modifications of the SM Ztt interactions, e.g. 4-fermion $\left(e^{+} e^{-} t \bar{t}\right)$ interactions. Current studies indicate that the effect of these operators cannot be disentangled from the physics modifying the $Z t t$ vertex by performing measurements at only one centre-of-mass energy. Several distant scales testing the $t \bar{t}$ process are required, and it is unlikely that a combination of the $t \bar{t}$ threshold and the slightly more energetic events at 365 $\mathrm{GeV}$ will suffice to probe the $Z t t$ vertex without model-dependent assumptions.

The right panel in Fig. 1, from Ref. [3], shows the results obtained from an EFT fit considering a restricted set of interactions, presented this time in a more traditional form, in terms of the $68 \%$ probability reach for the different Wilson coefficients or, equivalently, the NP interaction scale associated with each dimension- 6 operator. This is more convenient to connect the EFT results with specific models, via the matching of the model with the corresponding Wilson coefficients. In the next section we explore a few of these BSM interpretations of the EFT results. As the figure shows, the FCC-ee could indirectly probe scales up to several tenths of $\mathrm{TeV}$. 


\section{BSM interpretation}

The discovery of the Higgs boson at the LHC is key in the validation of the SM as a low-energy description of nature, but brings new questions related to the nature of this new particle and its interactions. For instance, we do not know whether the Higgs boson is a new elementary particle or a composite state. The latter possibility is explored in the so-called Composite Higgs models [7], which provide a solution to the hierarchy problem. These typically involve large deviations in the Higgs coupling which, according to the results established in the previous section, could be tested at permille-level accuracy in the FCC-ee Higgs factory. The FCC-ee reach for Composite Higgs models is illustrated in the top-left panel of Fig. 2, for a simplified scenario where the new dynamics is characterized by a single new coupling and heavy mass scale, $g_{\star}$ and $M_{\star}$, respectively [8]. The figure shows the great improvement in the indirect reach compared to that from the HL-LHC, as well as the individual impact of the different type of FCC-ee measurements.

One of the clear evidences of physics beyond the SM is provided by the observation of neutrino oscillations. This implies that neutrinos have mass, which is not possible within the SM. Such a mass, be it Dirac or Majorana, can be generated by enlarging the SM spectrum with additional neutrino-like fermions. In the standard seesaw scenario [9], Majorana masses can be generated through interactions with new heavy neutrinos transforming either as EW iso-singlets or iso-triplets. Even in the singlet case, the extra neutrinos can leave their imprint in precision measurements via mixing with the SM ones, e.g. modifying the decay of the muon, from which the Fermi constant is extracted. EW precision measurements can thus be used to set bound on the mixing angle with the SM neutrino flavours, $\theta_{e, \mu, \tau}$. Matching the results of the SMEFT fit to SM extensions with SM neutrino singlets [10] we find the following $1 \sigma$ limits:

$$
\left|\theta_{e}\right|^{2}<6 \times 10^{-5}, \quad\left|\theta_{\mu}\right|^{2}<6 \times 10^{-5}, \quad\left|\theta_{\tau}\right|^{2}<2 \times 10^{-4}
$$

where we have assumed that, for simplicity, only one of the mixings is allowed at a time. ${ }^{2}$

Another challenge of particle physics is explaining the origin of the matter-antimatter asymmetry in the universe. EW baryogenesis is a theory-compelling scenario where such asymmetry is linked to the nature of the EW phase transition [11]. For this to explain the observed asymmetry, however, NP beyond the SM is needed. In particular, it requires the EW symmetry breaking be a first-order phase transition, whereas the SM Higgs potential yields a second-order one. Models with extra neutral fields can help for this purpose. These type of particles are also common in models designed to address other important phenomenological questions, e.g. acting as portal to Dark Sectors, or to explain the hierarchy problem, as in the relaxion mechanism [12] or in models of neutral naturalness [13]. The simplest example of this type of scenarios with neutral particles are SM extensions with a real scalar singlet $\phi$ [14]. The scalar potential interactions allow this particle to mix with the Higgs, changing the Higgs couplings to the SM particles. From the results in Ref. [15], a one per-mile precision on the $H Z Z$ couplings, as expected from Fig. 1, would be able to explore most of the parameter space of the singlet extension leading to a strong first order transition. Other visible effects of the $H-\phi$ interactions include modifications of the Higgs self-interaction, which could be ${ }^{2}$ Similar results can be derived for the triplet case, where a richer pattern of effects arises, since the EW
properties of the charged leptons are also modified. The $1 \sigma$ limits that we find in this case are:

$$
\left|\theta_{e}\right|^{2}<9 \times 10^{-6}, \quad\left|\theta_{\mu}\right|^{2}<6 \times 10^{-5}, \quad\left|\theta_{\tau}\right|^{2}<10^{-5} .
$$


determined at the FCC-ee which $\sim 30 \%$ precision, via its contributions at loop level to single-Higgs processes [16].

The sensitivity studies discussed in the previous section are typically prepared assuming the absence of a NP signal, i.e. that the experimental measurements will match the SM predictions. While this is in general consistent with most of current observations, there are in fact some interesting anomalies dating back to the LEP era, which could point out to the existence of new particles testable at the FCC-ee. Such is the case of the forwardbackward asymmetry of the bottom quark, $A_{F B}^{b}$, roughly $2.5 \sigma$ below the SM prediction. The consistency of other measurements in the $b$ sector with the SM typically implies that any SM extension must protect the left-handed $Z b b$ coupling, while a significant contribution, $\sim 20 \%$, to the right-handed one takes care of explaining the observed value of $A_{F B}^{b}$. This discrepancy could be accommodated, for instance, in models with heavy vector-like quarks mixing with the bottom sector [17]. With a projected precision of the $Z b b$ vertex at the $10^{-3}$ level, see Fig. 1, the FCC-ee would be able to confirm whether such anomaly represents a real deviation from the SM. Moreover, the FCC-ee can offer additional insight of what kind of NP would explain this. For instance, if NP is largely modifying the couplings to the 3rd family quarks, the protection of the $Z b_{L} b_{L}$ vertex can follow from a custodial symmetry [18]. This, however, cannot simultaneously protect the $Z t_{L} t_{L}$ vertex, which would receive sizable corrections testable at the $365 \mathrm{GeV}$ run.

Also related to the physics of the bottom-quark sector, the FCC-ee can tell us about possible explanations of the recent flavour anomalies found in the $B$-physics sector at the LHC, notably in $b \rightarrow s \ell \ell$ processes. In particular, Leptoquark (LQ) extensions of the SM have received a renewed attention, as they could fit the experimental observations. The LQ couplings to the Higgs can contribute at the loop level to radiative $H$ decays and EWPO in a correlated manner that depends on the LQ model. While current constraints are not strong enough to test these correlations, the improved precision at the FCC-ee could be used to test these scenarios independently of the LQ couplings to fermions and help to distinguish between different LQ representations [19].

\section{FCC-ee sensitivity to new light degrees of freedom}

Despite the generality of the EFT approach presented in Sect. 2, this still relies on assumptions and it is therefore not fully model-independent. Of particular importance is to explore the possibility that new particle masses may not be significantly larger than the EW scale but, because of their properties, have escaped direct detection at the LHC. Such particles appear in many models of BSM physics and could be better searched for, directly or indirectly, at a future $e^{+} e^{-}$machine. In fact, some of the scenarios described in the previous section containing extra singlets or new sterile neutrinos fall in this category. In particular, if light enough, these type of particles could also appear in the $Z$ and $H$ decays, leading to extra contributions to the invisible branching ratios or, if the new particles decay back into the SM, to exotic decay channels. The clean environment of the FCC-ee would permit to tag such exotic channels, ${ }^{3}$ while the indirect (direct) determinations of the $H(Z)$ width, also possible only at an $e^{+} e^{-}$machine, would provide a strong bound on the total size of these non-SM decays.

${ }^{3}$ See [20,21] for direct sensitivity studies of such exotics $Z$ and Higgs decays at future $e^{+} e^{-}$colliders, where order of magnitude improvements with respect to the HL-LHC are possible in many cases. 

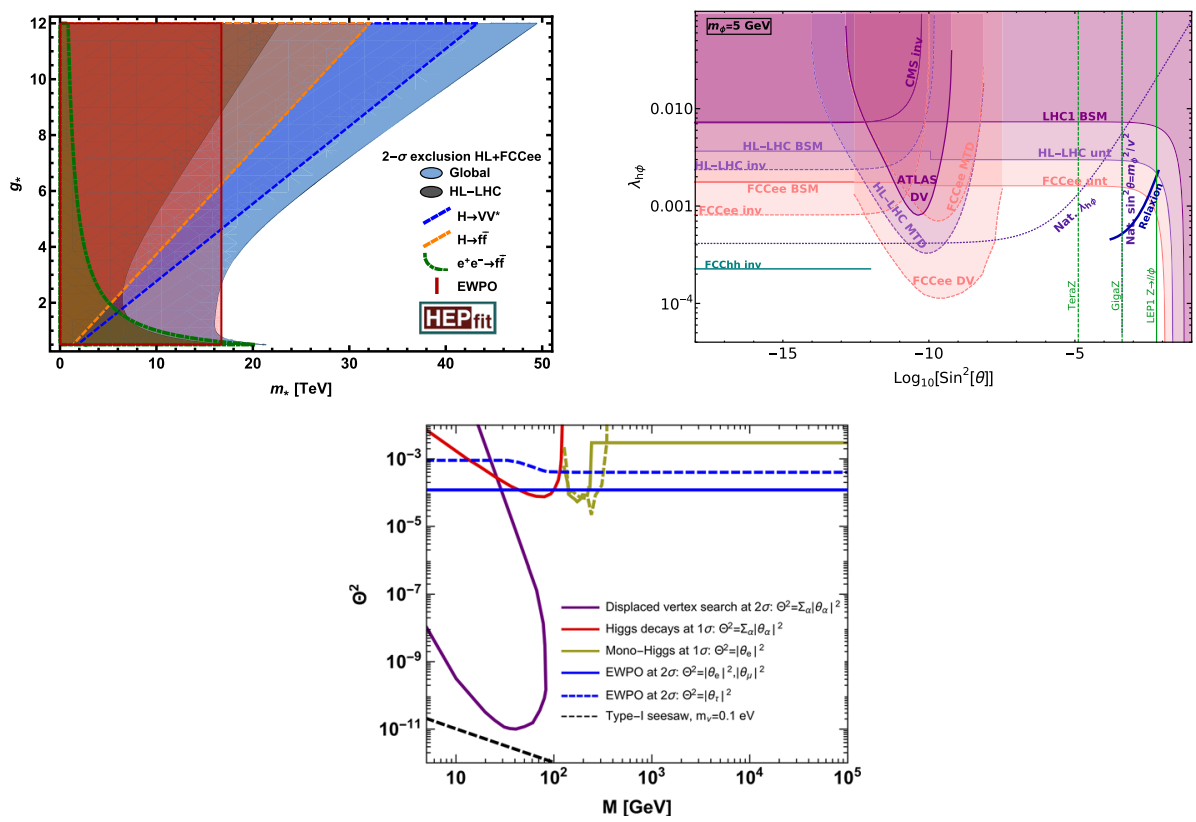

Fig. 2 (Top-Left) FCC-ee constraints on simplified composite Higgs scenarios parameterized by one NP coupling, $g_{\star}$, and mass scale, $m_{\star}$ (solid blue region). The bounds from different types of processes are overlaid on top, in transparent shades. For comparison, the dark shade in the background delimits the expected HL-LHC exclusion region. (Top-Right) From Ref. [23], constraints on the singlet extension of the SM in the $\lambda_{h \phi}$-sin $\theta$ plane. The portal coupling, $\lambda_{h \phi}$ only impacts the $H \rightarrow \phi \phi$ decays, while $\sin \theta$ controls the $\phi$ lifetime. Apart from the indirect bounds from Higgs decays and EWPO, the direct limits from, e.g. Displace Vertices (DV) are also shown. See [23] for details. (Bottom) FCC-ee sensitivity to the mass, $M$, of sterile neutrinos and their mixing, $\phi$ with the SM ones. (Adapted from Ref. [2,22], updating the limits from EWPO to match Eq. (2) at $2 \sigma)$

In the case of extra sterile neutrinos, if lighter than the $Z$, they could be directly searched during the $Z$-pole run, in the form of $Z \rightarrow v N$ decays. For the range of currently allowed mixing angles, $N$ is expected to have long lifetimes, leading to $Z$ decays containing displaced vertices. For these light new neutrinos, the $10^{12} \mathrm{Z}$ decays available at the FCC-ee would bring sensitivity to mixing angles as low as $10^{-11}$. A summary of the different probes of sterile neutrinos, both in the light and heavy regimes, is shown in the bottom panel of Fig. 2 .

The Higgs width, whose determination would only be possible at an $e^{+} e^{-}$machine, thanks to the measurement of the absolute $Z H$ cross section, would be a novel probe of models with light scalars singlets. The $H \rightarrow \phi \phi$ decays could manifest as invisible decays, displaced jets or untagged exotic decays, depending on the $\phi$ lifetime. With the FCC-ee precision, models leading to invisible (exotic) decays contributing with more than $0.2 \%(1 \%)$ to the total Higgs width would be ruled out at $95 \%$ probability $[3,23] .{ }^{4}$ The top-right panel in Figure 2, from the recent study in [23], summarizes the different types of constraints that would be possible with the FCC-ee measurements for a benchmark scenario with $m_{\phi}=5 \mathrm{GeV}$. As can be seen from the figure, a combination of indirect and direct searches at the FCC-ee would easily surpass the HL-LHC reach in the whole parameter space.

\footnotetext{
4 See also [24,25] for the implications of invisible and exotic Higgs decay searches at future colliders from the point of view of models where interactions with a light scalar singlet can induce a strong first-order EW phase transition.
} 


\section{Concluding remarks}

As we tried to convey, there are plenty of reasons that motivate a future $e^{+} e^{-}$collider if we aim to improve our knowledge of the EW sector and learn about NP which could be hiding as small corrections to SM processes. These go from the remarkable improvement in the precision with which we know some of the particle properties today, to the access to measurements that, so far, seem only possible in the clean environment of a $e^{+} e^{-}$machine, and that would give access to properties the LHC cannot test, such as the Higgs width. These different probes will be crucial not only in testing the structure of nature to scales far beyond the LHC reach, but also in exploring the "holes" in the BSM space that the LHC, as a hadronic machine, is limited to probe.

We conclude by reminding that the BSM search strategy discussed here is based on the pessimistic, though at this point likely, assumption that nothing new will be found at the LHC. Obviously, any new discovery would completely change the focus of searches at future colliders, increasing the importance of indirect precision tests. The consistency of such tests with the presence of new particles could not only tell us about the properties of such states, but also give indirect information about the existence of other particles, exactly in the same way that LEP/SLC precision tests told us about the Higgs before this was discovered at the LHC. At any rate, with positive or negative LHC NP findings, it is clear that the FCC-ee is a necessary step not only towards learning about the properties of the SM particles but also for the purpose of guiding direct searches at a future $100 \mathrm{TeV} p p$ machine.

Funding Open Access funding provided thanks to the CRUE-CSIC agreement with Springer Nature. This project is co-funded from the European Union's Horizon 2020 research and innovation programme under grant agreement No 95175 .

Open Access This article is licensed under a Creative Commons Attribution 4.0 International License, which permits use, sharing, adaptation, distribution and reproduction in any medium or format, as long as you give appropriate credit to the original author(s) and the source, provide a link to the Creative Commons licence, and indicate if changes were made. The images or other third party material in this article are included in the article's Creative Commons licence, unless indicated otherwise in a credit line to the material. If material is not included in the article's Creative Commons licence and your intended use is not permitted by statutory regulation or exceeds the permitted use, you will need to obtain permission directly from the copyright holder. To view a copy of this licence, visit http://creativecommons.org/licenses/by/4.0/.

\section{References}

1. A. Abada et al., Eur. Phys. J. C 79(6), 474 (2019)

2. A. Abada et al., Eur. Phys. J. ST 228(2), 261-623 (2019)

3. J. de Blas et al., JHEP 01, 139 (2020)

4. J. de Blas, G. Durieux, C. Grojean, J. Gu, A. Paul, JHEP 12, 117 (2019)

5. A. Blondel, A. Freitas, J. Gluza, T. Riemann, S. Heinemeyer, S. Jadach, P. Janot, [arXiv:1901.02648 [hep-ph]]

6. P. Janot, JHEP 04, 182 (2015)

7. R. Contino, Y. Nomura, A. Pomarol, Nucl. Phys. B 671, 148-174 (2003)

8. G.F. Giudice, C. Grojean, A. Pomarol, R. Rattazzi, JHEP 06, 045 (2007)

9. R.N. Mohapatra, Phys. Rev. Lett. 56, 561-563 (1986)

10. J. de Blas, J.C. Criado, M. Perez-Victoria, J. Santiago, JHEP 03, 109 (2018)

11. G.W. Anderson, L.J. Hall, Phys. Rev. D 45, 2685-2698 (1992)

12. P.W. Graham, D.E. Kaplan, S. Rajendran, Phys. Rev. Lett. 115(22), 221801 (2015)

13. Z. Chacko, H.S. Goh, R. Harnik, Phys. Rev. Lett. 96, 231802 (2006)

14. D. O’Connell, M.J. Ramsey-Musolf, M.B. Wise, Phys. Rev. D 75, 037701 (2007)

15. P. Huang, A.J. Long, L.T. Wang, Phys. Rev. D 94(7), 075008 (2016) 
16. M. McCullough, Phys. Rev. D 90(1), 015001 (2014) [erratum: Phys. Rev. D 92(3), 039903 (2015)

17. D. Choudhury, T.M.P. Tait, C.E.M. Wagner, Phys. Rev. D 65, 053002 (2002)

18. K. Agashe, R. Contino, L. Da Rold, A. Pomarol, Phys. Lett. B 641, $62-66$ (2006)

19. A. Crivellin, D. Mueller, F. Saturnino, [arXiv:2006.10758 [hep-ph]]

20. Z. Liu, L.T. Wang, H. Zhang, Chin. Phys. C 41(6), 063102 (2017). [arXiv:1612.09284 [hep-ph]]

21. J. Liu, L.T. Wang, X.P. Wang, W. Xue, Phys. Rev. D 97(9), 095044 (2018)

22. S. Antusch, E. Cazzato, O. Fischer, Int. J. Mod. Phys. A 32(14), 1750078 (2017)

23. E. Fuchs, O. Matsedonskyi, I. Savoray, M. Schlaffer, [arXiv:2008.12773 [hep-ph]]

24. M. Carena, Z. Liu, Y. Wang, JHEP 08, 107 (2020). https://doi.org/10.1007/JHEP08(2020)107

25. J. Kozaczuk, M.J. Ramsey-Musolf, J. Shelton, Phys. Rev. D 101(11), 115035 (2020) 